\title{
Un (an)alfabeto d'amore
}

Pubblichiamo qui una seric di sonetti inediti dovuti alla penna di Francesco Maria Paglia. Il nome non è fra i piú familiari agli studiosi di letteratura italiana, ma lo è ai musicologi. Il nome di Paglia è infatti legato alla cultura napoletana della fine del secolo XVII ed alla fama di Alessandro Scarlatti, per il quale compose numerosi libretti. Ricordiamo: La Didone delirante (1696); Commodo Antonino (1696); L'Emireno o vero il Consiglio dell'Ombra (1697), opera drammatica; Mutio Scevola (1698); Il prigioniero fortunato (1698); Dafni (1700), favola boschereccia; Il pastore di Corinto (1701), opera pastoralc.'

Spesso il suo lavoro era di collaborazione o anche di rimaneggiamento: in quest'ultima categoria rientra la "facile" riclaborazione di Gl'Inganni felici, un libretto di Apostolo Zeno basato su materiale ricavato da Erodoto, a cui Paglia aggiunse dei personaggi buffi. Scrivono al riguardo Pagano e Bianchi:

Scomodare Erodoto per la trama di una commedia era impresa da pedante; ma a Napoli, secondo il solito, l'abate Paglia, o qualche altro poetucolo da teatro, mandò in fumo l'impegno classico del futuro poeta cesareo, inserendo nel "drama" i consueti due personaggi buffi. (155)

Il 19 Dicembre, sempre al San Bartolomeo, andava in scena la Semiramide del Paglia, con la musica di Giuseppe Aldrovandini; ed il 27 il nostro librettista "si presentava con un dramma originale, Cesare in Alessandria, messo in musica dallo stesso Aldrovandini, uno scapestrato compositore di talento" (156).

A questa ricca produzione librettistica si aggiunge una vastissima collezione di "Serenate e grandi cantate d'occasione," come Venere, Adone e Amore, serenata a tre voci e strumenti, e le numerose "Cantate a una voce, strumenti e basso continuo," come "Ben mio, quel verme alato," e "Là dove al sonno in braccio," tutte composizioni musicate da Alessandro Scarlatti. ${ }^{2}$

Del tutto sconosciuta c̀ la sua produzione poetica. Questa, o almeno una parte di questa, si trova raccolta in un manoscritto conservato 
presso la Biblioteca Angelica di Roma, manoscritto che dai fregi e dagli emblemi sembra allestito per la stampa, ma da quanto risulta non fu mai pubblicato. È una produzione varia di sonetti, canzoni, canzoni per musica e componimenti lirici, perfino in spagnolo. Il manoscritto figura nel catalogo degli Inventari dei manoscritti delle Biblioteche d'Italia, opera fondata da Giuseppe Mazzatinti, al volume numero LXXVI, pagina 109, numero 2318. Il manoscritto ha per titolo Rime varie di Fran. ${ }^{c o}$ M. ${ }^{a}$ Paglia, e contiene le seguenti raccolte:

\section{Sonetti amorosi;}

Capricci;

Sonetti eroici;

Sonetti sacri;

Canzoni;

Componimenti per musica;

Rimas españolas.

È un manoscritto cartaceo, del XVIII secolo, di 490 pagine numerate e 32 non numerate, ed è cosí descritto nel catalogo:

"Frontespizio con disegno a penna e antiporta, pure disegnata a penna, raffigurante Apollo e le nove Muse. Leg. coeva in pelle con fregi in oro sul dorso."

Le figure sono disposte a piramide, con Apollo, dio della poesia, al centro in alto, circondato dalle Muse. Il tema amoroso è già implicito in questa scena boschiva e ariosa, senza didascalie, e si continua e precisa alla pagina seguente, ancora con il motivo di foglie e fronde, dove il titolo della raccolta, Rime varie di Frant. ${ }^{c o} M^{a}{ }^{a}$ Paglia, figura come un libro aperto come un cuore alla ferita d'Amore e trafitto dalla freccia del dio, con la didascalia "Vulnerat, et scribit"; in basso, a sinistra, Amore alato che dorme, nudo e rubicondo, con la faretra vicina, per terra, e l'iscrizione "Vulnerat, et dormit."

Una rapida scorsa a questo voluminoso manoscritto rivela un poeta mediocre non per mancanza di tecnica artistica, ma per l'immaginazione. Tuttavia fra questi componimenti è notevole la prima raccolta, intitolata Sonetti amorosi, che sono in tutti ventuno, come le lettere dell'alfabeto, oltre al sonetto introduttivo: queste poesie trovano la loro unità in una tecnica specifica che è il lipogramma.

I componimenti lipogrammatici non rappresentano una novità nel Seicento, ma per quanto io sappia non esiste una collana simile. Il li- 
pogramma era sempre limitato alla prosa; questo espediente retorico ha origini antiche ed esiste sempre dove il poeta preferisce l'affettazione alla naturalezza; può inoltre esistere semplicemente a livello verbale o combinare concetti o parole. Il lipogramma consiste nel sopprimere sistematicamente una lettera dell'alfabeto, un fonema da un testo: l'esempio piú antico risale al maestro di Pindaro, il pocta e musico Laso (metà del VI secolo a.C.), che scrisse poesie senza il sigma. Nestore di Laranda costruí un'lliade in cui ogni libro mancava di una lettera dell'alfabeto; cosí fece Trifiodoro per l'Odissea. In questa storia del lipogramma si ricorda anche il nome di Fabio Planciade Fulgenzio, che impiegò questo artificio nel suo De aetatibus mundi et hominis; e si giunge cosí alla Spagna del XVII secolo, con una composizione lipogrammatica alla conclusione del romanzo picaresco Estebanillo González.

Il lipogramma è un ricorso retorico opposto a quello del tautogramma, o verso pangrammatico, che consiste nell'iniziare piú parole possibili con la stessa lettera, una forma di allitterazione esasperata, come nel famoso verso di Ennio:

O Tite, $t$ ute, $T$ at $\mathrm{i}, t$ ibi $t$ ant a, $t$ yranne, $t$ ulist i. (Curtius 283)

Mentre il pangramma è una figura di "presenza," il lipogramma è una figura di "assenza" (Pozzi 106). ${ }^{4}$

I Sonetti amorosi di Francesco Maria Paglia che qui pubblichiamo hanno qualcosa di paradossale: utilizzano il lipogramma, uno degli aspetti piú arditi ed insieme facili della tecnica baroccheggiante, ma per quel che riguarda gli aspetti concettisti della tecnica barocca c'è ben poco. Le immagini, le iperboli sono tutt'altro che ardite, e si avverte dappertutto una musicalità facile ed un metaforeggiare di stampo prearcadico. Forse questa levità di contenuti c di immagini è dovuta al fatto che Paglia componesse per musica.

Poiché mancano dati interni ed esterni per datare questi sonetti, è difficile stabilire in che periodo della sua attività Paglia li abbia composti, ed è arduo capire in che linea culturale li si possa collocare. Forse non sarebbe sbagliato vedere in questa poesia, per la sua elocutio alquanto dimessa rispetto a quella del pieno Barocco napoletano, un risultato o documento dell'avanguardia di Leonardo Di Capua.

I Sonetti amorosi portano come emblema, sul frontespizio della collana, la faretra e le frecce, reiterazione del tema amoroso, e sono 
dedicati come un galante alfabeto d'amore con questa dicitura:

Si dedica all'Ill. ${ }^{\text {ma }}$ Sig. ${ }^{\text {ra }}$ D. Angela Voglia il seguente Alfabeto de' Sonetti, a ciascuno de' quali mancano alcune lettere. - A te che sei de le mie rime impresa.

Questa dedica spiega in modo essenziale i poli del fare poetico di Paglia. Come tutti i poeti egli compie un' "impresa" amorosa, che costa "sudori" poetici, e la trascrive con i segni dell'alfabeto, con le grammata: ma il poeta è impari all'impresa che intraprende, per cui il suo alfabeto sarà sempre incompleto, ed il canto della sua donna quasi sempre approssimativo; l'amore non sarà mai pieno o ricambiato, e l'espressione del poeta può avere piú del balbettio che della lucidità poetica: cosí si chiude il sonetto dedicatorio:

Canto, o bella, d'amor, ma nol comprendo,

e benché avvezzo a l'amorose scuole

l'alfabeto d'amore io non intendo.

Il lipogramma è infatti, come sappiamo, una figura di assenza; pertanto il significato di una collana lipogrammatica può essere questo: nessun momento dell'amore per la donna è completo dall'alfa all'omega. L'amore non si realizza perché è ineffabile e la sua instabilità si riflette e rientra nel gioco dell'alfabeto, come indice di mancanza di integralità. C'è tuttavia un'unità che è frammentata in ventuno unità, in cui le immagini si accavallano, si ripetono e si contraddicono.

La mancanza di integralità viene espressa da un bisogno di accumulazione, ed il sonetto conclusivo, Moralità d'amante, sarà tutto tramato sul concetto reiterato di una "mancanza". L'amore non si conoscerà mai integralmente, come sostanza, ma se ne possono elencare tutti gli accidenti. La collana diventa cosí una sorta di summa amorosa, che finisce col dare piú un'idea dell'amore che la storia di un amore vero. A veder bene, questo sistema distanzia ulteriormente il poeta dalla donna, in quanto questa finisce con l'essere piú un'idea risolta in molti fenomeni che una forza di energia sentimentale.

Il poeta è consapevole di questa situazione quando canta nel sonetto introduttivo alla collana successiva, Capricci:

Già leggesti i mici carmi, ove scolpita

si rimira l'idea di varij amori;

a nuove fole o bella ora t'invita

Genio, che ti consacra i suoi sudori. 
In questo modo il lipogramma non è solo una figura verbale, ma una figura a livello di concetto; questi versi sembrano indicare, inoltre, una chiara linea di continuità fra le due raccolte, un ancllo di congiunzione sul piano pratico e su quello tematico.

In tutta la gamma dei "varij amori" è sempre sottolineato il senso di una presenza-assenza, un'impossibilità di completezza per la storia d'amore, come incompleti sono i carmi del poeta che mancano di lettere, "come mancan le lettere ai mici carmi". La casistica di un amore sempre provvisorio ed incompleto è tutta risolta e stemperata in questo "alfabeto d'amore," nel tessuto verbale: di questo alfabeto il poeta cerca vanamente di decifrare e possedere tutti i segni.

La spia di una mancanza d'amore è già nell'artificio della "finzione" espressa nel sonetto di dedica:

Non ho, benché la fingo, un'alma accesa, soggetta a le follie di varij amori.

Siamo soltanto all'inizio, ed è già come una dichiarazione programmatica.

Il poeta conosce "d'altrui gl'affanni," ma non riesce a decifrare i segni dell'"alfabeto d'amore," con la similitudine del fanciullo che apre gli occhi alla luce "e non conosce il sole." Questo dialogo continuo ed asasperato con l'Amore personificato, forte presenza di concetto, si svolge come sulla scena, con tutti i caratteri di teatralità di una storia d'amore:

Onde Amore in due parti il cor divide,

e teatro è il mio seno, ove due strali

fanno a chi piú m'accende, a chi m'uccide

(Amor doppio).

A te che sei de le mie rime impresa, a te che degna sei di mirti e allori, di cetra polverosa un tempo appesa brevi corde consacro, e lunghi errori:

cosí canta nel sonetto dedicatorio, in cui "impresa" e "mirti e allori" segnano la sproporzione tra l'effimero tema d'amore trattato ed il presunto impegno epico del poeta.

Fitte sono anche le interrogazioni retoriche, che in realtà sottendono un'ansia solo letteraria:

Dimmi, o Nume bendato, a crudi eventi serbar sempre vorrai l'alma ferita? 
Godrà l'anima mia giamai contenti,

e incontro a tanti affanni, havrò mai vita?

Tu taci, ingrato; e ogn'or dovrò soffrire

l'avvelenato ardor de le tue faci, barbaro, arciero dio, ma non gioire?

Cosí tratti, o crudele, i tuoi seguaci?

(Oracolo d'amore)

Già nei titoli dei sonetti è presente la varia gamma della casistica amorosa, dall'incostanza alla fedeltà: l'Amore è "impossibile, geloso, lontano, loquace, tacito, doppio, virtuoso, costante, vario, corrisposto." I "varij amori" rappresentati su questo palcoscenico (non a caso Paglia scriveva per la scena) sono dolorosi e negativi: Amore "è un mal, che piú si fa gemello / a chi piú gli s'appressa" (Amor vario), un "gran tiranno" (Amor corrisposto); il tutto è tramato sul tema, insistito e ricorrente, della lontananza e dell'assenza, di un'impossibilità d'amore già presente nel primo sonetto, Amore impossibile:

Su i diletti e i tormenti offeso e scosso,

o corrisposto o no, lungi o vicino, io posseder l'idolo mio non posso.

Un'altra costante tematica è la separazione, come in Partenza:

Addio, mio core, addio. Venne quell'ora,

che da' tuoi lumi io viverò lontano

La seconda terzina riprende in ciclo, nell'ultimo verso, l'endecasillabo d'apertura:

Questa ne le mic pene al cieco dio

mercede io chiederei di mia costanza;

addio, mio core, addio, mia vita, addio:

versi in cui la teatralità di quell' "addio" ripetuto e della lontananza che divide è scandita dall'insistenza dell'asindeto, figura comunissima ed efficace in questi sonetti.

L'amore positivo è nella costanza dell'amante: l'ultimo sonetto chiudcrà il ciclo con la "moralità dell'amante" ormai piú maturo e la reiterazione di una "mancanza," quasi ossessiva già nell'anafora dei cosí nella similitudine d'apertura:

Come manca la rosa in seno al prato, cosímancano l'ore al viver mio; 
cosi perde lo stral l'Arciero ingrato, cosi manca lo scopo al cicco dio.

Mancan gli spirti piú vivaci, e chiusa

Guerra, guerra ad Amor, mio core a l'armi; già per lui manca l'estro a la mia Musa, come mancan le lettere ai miei carmi.

(Moralità d'amante)

Questa analisi in superficie del sentimento amoroso origina giochi di parole a livello formale, sintattico e semantico; frequente, per fare un esempio di questo gusto retorico, l'anafora, figura di ripetizione, come nel sonetto di dedica:

A te che sei de le mie rime impresa, a tc che degna sei di mirti e allori

Molto frequente è l'asindeto, a creare sospensione e distanza, uno stacco:

Stragi, morti, ruina, io bramo, io voglio

scordo, abborrisco i suoi passati onori, stragi, morti, ruina, armi, battaglia.

Paci non bramo piú, voglio rigori; asta, spada, lorica il cor m'abbaglia, lascio Amor, voglio Palla, amo gl'allori.

(Lascia Amore)

A volte occupa tutta una terzina, un lungo elenco:

Guerra, nodi, catene, ingiurie, affanni, schiavitú, tirannia, noia, tormento, rischi, angoscia, timor, cadute, inganni:

è la casistica della negatività d'Amore nel sonetto Che sia Amore.

Degna di nota è anche l'anastrofe, che inverte l'ordine normale, come nel verso

Ciò che nel cor si porta in van si fugge

(Allontanarsi per non amare);

e la ripetizione, per ben due quartine, che citiamo per intero:

Quante stagioni ho pianto, e quante ho spese seguendo un occhio nero, un aureo crine; quante saette in sen mi son discese, quante ferite mai, quante ruinc. 
Quanti sonni ho perduti, e quante offese ho provato in amore, e quante spine per due guancie di rose, e quante accese fiamme ho sofferto; e che ritrassi in fine?

(Che sia Amore)

Si rivela importante, per l'efficacia dell'interrogazione conclusiva, anche l'uso dell'enjambement in "e quante accese/fiamme ho sofferto."

Un'analisi piú attenta metterebbe in luce con facilità astuzie retoriche affini a quelle sottolineate. Nel complesso esse dimostrano, nel nostro poeta, una perizia non disprezzabile, un mesticre consumato: anche a questo livello la coerenza interna dei Sonetti amorosi viene cosí confermata.

Passiamo ora a trascrivere questo testo inedito. Ogni sonetto occupa una pagina del manoscritto, per un complesso di ventun pagine (dalla pagina 3 alla 24), dall'A alla Z. Si riportano anche, per facilitare al lettore la comprensione della collana lipogrammatica, il sonetto introduttivo ( $A$ te, che sei de le mie rime impresa) e quello di dedica della seconda raccolta, Capricci, per evidenti legami tematici (Già leggesti i miei carmi, ove scolpita / si rimira l'idea di varij amori).

Nel trascrivere questi sonetti ci siamo limitati ad apportare leggeri ritocchi, intervenendo solo per quel che riguarda la punteggiatura e l'aggiunta degli accenti dove l'uso moderno li richiede. Intatta si conserva la grafia di termini arcaici come labra, della congiunzione composta fin che, di avverbi come a bastanza, in fine, ogn'ora, tal'or, o di preposizioni del tipo de la, de l', per fedeltà alla cadenza del testo originale. Le inutili maiuscole sono state eliminate. Si è conservata invece l' $h$, anche quella etimologica, dato che la natura lipogrammatica della collana non ne consente la soppressione, essendo anche l'h una delle lettere dell'alfabeto.

Per ogni sonetto viene rappresentata al margine a sinistra in alto la lettera dell'alfabeto mancante, dall'A alla Z; alla destra del titolo sono indicate altre lettere mancanti, ma che non indicano evidentemente soppressioni sistematiche. In pochi casi, per le condizioni del manoscritto, queste ultime risultano di difficile lettura, e in ogni modo non sembra necessario decifrarle con matematica cer- 
tezza, poiché non indicano alcun sistema. Si noterà che dopo la $u$ viene la $x$ invece della $v$, dato che la $v$ aveva la stessa grafia della u: e questo per completare le ventun lettere dell'alfabeto. Ho inoltre unificato la numerazione dei sonetti, che nel manoscritto portano le cifre arabiche all'inizio e poi quelle romane.

Anche se in alcune istanze, pochissime per la verità, il testo non è perspicuo, si è potuto renderlo chiaro con leggeri interventi sulla punteggiatura, e non sembra offrire al lettore cruces particolari.

Loyola University of Chicago

\section{SONETTI AMOROSI}

Si dedica all'Ill. ma Sig. ${ }^{\text {ra }}$ D. Angela Voglia il seguente Alfabeto de' Sonetti, a ciascuno de' quali mancano alcune lettere

A te, che sei de le mie rime impresa, a te, che degna sei di mirti e allori, di cetra polverosa un tempo appesa brevi corde consacro, e lunghi errori.

Non ho, benché la fingo, un'alma accesa soggetta a le follie di varij amori; a bastanza provai del cor l'offesa, né di vane speranze ammetto ardori. Canto d'altrui gl'affanni, e come suole avvenir a un fanciul, che gl'occhi aprendo vede la luce, e non conosce il sole, canto, o bella, d'amor, ma nol comprendo, e benché avvezzo a l'amorose scuole l'alfabeto d'Amore io non intendo.

A. Amore impossibile B. Q. X. Z.

Voi che feriti in mille forme e mille per un volto divin perdeste il core, 
le vostre dite pur, che son scintille presso il foco, ond'il mio si strugge e more.

Cor, che provi le pene, oh Dio tu dille, dolce se m'offuscò fu lo splendore; io vissi e vivo prigionier di Fille, e pur il genio mio non fece errore.

Scopo d'un crudo e perfido destino con freno e sprone, e ritenuto e mosso, morte non veggio, e chi m'uccide inchino.

Su i diletti e i tormenti offeso e scosso, o corrisposto o no, lungi o vicino, io posseder l'idolo mio non posso.

B. Partenza F. G. X.

Addio, mio core, addio. Venne quell'ora che da' tuoi lumi io viverò lontano; ma non vivrò poiché la nuova Aurora chiamerà il Sol senza di loro invano.

Di viver sol potrei sperare all'ora, quando il tuo cor, de' sensi miei sovrano, meco venisse, e meco stasse ancora in mar, in terra, al rivo, al monte, al piano.

Questa sola o mio Nume è la speranza per cui qualche sollievo al petto mio concederà tal'or la lontananza.

Questa ne le mie pene al cieco dio mercede io chiederei di mia costanza; addio, mio core, addio, mia vita, addio.

\section{3}

C. Amor geloso

B. H. X.

Amo donna gentile, e la speranza del mio gioir non è del tutto estinta; non tormenta il pensier la lontananza, e ne men dal disprezzo è l'alma avvinta.

Peno per essa, è ver, ma non avanza il diletto la pena, onde se vinta la speme è dal timor, tutta arroganza torna dentro al mio seno, e non è finta.

Dunque goder dovrei, se il dio d'amore 
sí tiranno non è ne l'alma mia,

c non posa* per essa il suo furore.

Ma pur non $\grave{e}$, quale il desir vorria, mentre l'empio riserva ogni vigore ne l'eterno martir di gelosia.

* Ricostruisco cosí, ma è di difficile lettura nel ms.

4

D. Amor lontano

C. Q. X.

Amai beltà pietosa, e amato fui senza soffrir né falsità, né inganno; e il Pargoletto sí tiranno altrui ne la ferita mia non fu tiranno.

Piansi, nol niego, e fui soggetto a lui, ma fu bello il martir, grato l'affanno; mentre vagheggio un Nume, e i raggi sui ben gli veggon le sfere, ma non gl'hanno.

Vissi lunga stagion non lieto appieno, ma non vissi giamai senza speranza, latte, ristoro, et alimento al seno.

Toltami al fin l'amabile sembianza, mi sparge un fiero, e barbaro veleno ogn'istante al pensier la lontananza.

5

E. Amor loquace

D. X. Z.

In ogni altar, che a gl'olocausti aspira, piacciono, quanto il cor, labra loquaci; tacciano i Numi sol, taccia la pira, la vittima non già tra fumi, o faci.

Al sacrificio ogn'or taccia chi mira, ma chi brucia non può; troppo vivaci vibra gli strali Amor; parli chi spira; troppo vil morirai; cor mio, tu taci?

Potrà Lilla far sí ch'io non la miri, ma non può mai col suo tiranno orgoglio proibir al mio cor pianti, o sospiri.

Soffrirà l'amor mio scritto in un foglio, già son troppo giganti i suoi martiri; mi scacci Lilla, o no; parlar io voglio. 
6

F.

E. H. Q. X.

Non piú d'Amor andar vogl'io tra l'armi:

stragi, morti, ruina, io bramo, io voglio; di mia vita arrossisco, anzi già parmi provar su l'alma un immortale orgoglio.

Mai piú da un biondo crin miri allacciarmi Cupido, o rida a l'aspro mio cordoglio; non piú la lira mia stimoli i carmi a ridir la cagion di cui mi doglio.

Amor a l'alma mia piú non s'uguaglia, scordo, abborrisco i suoi passati onori, stragi, morti, ruina, armi, battaglia.

Paci non bramo piú, voglio rigori; asta, spada, lorica il cor m'abbaglia; lascio Amor, voglio Palla, amo gl'allori.

\section{7}

G.

Torna ad Amore

B. H. Q. X.

Perdona, Amor, perdona; ecco mi pento; la tua pietà, la tua clemenza invoco; vale piú d'un piacere un tuo tormento, piú d'un Zefiro è a me caro il tuo foco.

Per te senz'alma, e senza cor mi sento, ma poi senza di te non trovo loco; non v'è pace per me, non v'è contento, nere sono le stelle, e il sole è poco.

Torno a le tue catene, e se la sorte a penar per un volto il core invita, annodi il petto mio laccio piú forte.

Vanne pure ai diletti anima ardita, va' per Amore ad incontrar la morte, pria di passar senza di lui la vita.

\section{8}

H.

Temo, e non amo; e se tal volta Amore tenta mostrar un dolce nodo al seno, tremo, pavento, e provo all'or nel core, senza provarlo ancora, un gran veleno. 
Folle parlo tra me: questo ¿̀ dolore?

È tema, o no? fosse speranza almeno; speranza no: dunque cos'è? terrore del mal d'amor; penso, non amo, e peno.

Rara forza d'un Nume! aspro, o clemente, sempre è temuto; e pur l'alma seguace è per esso gelata, e a un tempo ardente.

Non so se Amor con me vuol guerra, o pace; non lo conosco ancor, pena la mente sol temendo l'ardor de la sua face.

Quando Amor saettò l'alma nel petto tacque l'alma l'offesa, e tace ancora; e se novello mal prova ad ogn'ora la causa tacerà del crudo affetto.

Espero torna con funesto aspetto, Fosforo va con la nascente Aurora, uno le cose occulta, uno le onora, e non paleso l'adorato oggetto.

Cerca sempre mercede amor loquace, ma celar fra me stesso ogn'or pretendo a l'arco, ond'è scoccato, e dardo, e face. Speme non vuo', ma vuo' tacer morendo, e remora al godere Amor se tace, segno d'una grand'alma è amar tacendo.

Quante stagioni ho pianto, e quante ho spese seguendo un occhio nero, un aureo crine; quante saette in sen mi son discese, quante ferite mai, quante ruine.

Quanti sonni ho perduti, e quante offese ho provato in amore, e quante spine per due guancie di rose, e quanto accese fiamme ho sofferto; e che ritrassi in fine?

Guerra, nodi, catene, ingiurie, affanni, schiavitú, tirannia, noia, tormento, rischi, angoscia, timor, cadute, inganni. 
E questo è Amore? oh perfido momento che mi fe' tuo seguace, e giorni, ed anni, se un tuo frutto verace è un pentimento.

M. Allontanarsi per non amare

Q. X. Z.

Fuggo, e dove, e chi fuggo? e chi difende da la forza d'un dio le vie del core?

Chi fuggir può fato crudel, che offende, et indora il dolor col suo splendore?

De l'aspra face ria che in ciel s'accende chi estinguer può l'avvelenato ardore? Chi consola il pensiere, e chi l'intende se il pensier di se stesso è traditore?

E chi fuggo? le stelle, onde deriva genio fatal d'una beltà, che strugge, che diletta, ed affanna, uccide, e avviva.

È troppo dolce il nettare, che sugge, e benché de la vista il cor si priva, ciò che nel cor si porta in van si fugge.

Mira [Amor mi dicea]; vedi quel viso?

Il veggio [io dissi]; [ci replicò] ti piace?

Sí [gli risposi]; [ed ei] che paradiso!

Amalo; va'[diss'io]; voglio la pace.

Mira [dicea], su 'l ciglio ha il sol diviso; ov'è ascosa [diss'io] fiamma vorace; su le gote [dicea] la sede ha il riso; ivi cela [risposi] ardori, e faci.

Che temi? [replicava]; [io] che m'uccida; chi muor per me? [rispose]; [io dissi] è morta l'alma di chi di te crudel si fida.

Il laccio mio [dicea] ristoro apporta; quel volto [replicai] d'Amore è guida, guida di cieco a le cadute è scorta. 
O.

Seguí la mente insana infante Nume fin che l'età trattenne i passi alati; ma appena ai disinganni aperse il lume pianse l'età presente, e $\mathrm{i}$ dí passati. Già d'un'idea piú eccelsa erge le piume fissa di gran pianeta ai vanni amati; e assai di sé l'anima mia presume se di luce divina ha i raggi armati.

Pugnan piú genij in lei, ma in lei pur viene il desio di cercar la prima calma, e di franger per sempre empie catene.

Ama, ma che? de la virtú la palma; ama, e che? di fuggir perigli, e pene; ama l'alma, ma che? la pace a l'alma.

\section{4}

P.

\section{Oracolo d'Amore}

Q. X. Z.

Dimmi, o Nume bendato, a crudi eventi serbar sempre vorrai l'alma ferita? Benché solo temer dovrei tormenti dove un cieco tiranno il core invita.

Godrà l'anima mia giamai contenti, e incontro a tanti affanni, havrò mai vita?

Sciogli fanciullo dio liberi accenti, né sembri a te la mia richiesta ardita.

Tu taci, ingrato; e ogn'or dovrò soffrire l'avvelenato ardor de le tue faci, barbaro, arciero dio, ma non gioire?

Cosí tratti, o crudele, i tuoi seguaci?

[Disse l'idolo al fin] Vanne a morire, Clori non è per te, soffrilo, e taci.

Q.

Due deità sovrane invia Cupido per far nobile insulto a l'alma forte; or fra due belle labra ci cerca il nido, or fra due luci belle apre le porte. 
A chi di loro anima mia ti fido?

Con le luci, o coi labri havrai piú sorte?

Vacillante pensier di te diffido;

dove la vita avrai, dove la morte?

Son le luci di Fille armi immortali, son le labbra di Clori urne omicide, fulmini vivi, e porpore fatali.

Onde Amore in due parti il cor divide, e teatro è il mio seno, ove due strali fanno a chi piú m'accende, a chi m'uccide.

\section{6}

R.

$$
\text { Amor virtuoso }
$$

H. X. Z.

Amo, e speme non seguo; amo un oggetto amabile in se stesso, e l'alma amante pasce nel bello un innocente affetto, né domanda pietà da quel sembiante.

Non avido di baci, amo l'aspetto, amo la bocca, e il ciglio fulminante contemplo, ma del foco in me concetto aita mai non voglio, e son costante.

Piú del volto amo l'alma, e in me non desta quello un genio lascivo, onde non svelo a lei fiamma gentil, se non molesta.

In me di gelosia non sento il gelo;

e di quello il mio seno ama piú questa, se in quello il mondo s'ama, in questa il cielo.

\section{7}

S.

\section{Amor costante}

Q. X. Z.

Amare, e ritrovar nel bene amato

il premio, e la mercé, che amore attende, porta il nome d'Amor, ma non accende con oggetto di gloria un cor ben nato.

Ma l'amar fedelmente un petto ingrato, che vittime non cura, o non intende, c̀ una fiamma immortal, che l'alma offende, è il trionfo maggior d'un cor piagato.

Tal'io, che adoro voi lumi tiranni, e mi pregio, che il cor già non m'è tolto dal piacer, che la mente empie d'inganni. 
Onde rimproverar vogl'io rivolto a colei, che credea di darmi affanni; vale piú l'amor mio, ch'il tuo bel volto.

Gran chimera è la fede: e dove apprese l'alma ad amar una sol cosa al mondo! Perché nudrir non può le voglie accese di ciò, ch'è vago, amabile, e giocondo. Perché non deve un cor provar l'offese d'un bel ciglio ch'è nero, e un crin, ch'è biondo; perché ad un bianco sen far piú difese, e ad un labro soave, c rubicondo!

Vani inganni d'un cieco: acciò maggiore sia la pena de l'alma, ogn'or novello ne le leggi si rende, e cangia errore. Amore è un mal, che piú si fa gemello a chi piú gli s'appressa, e al fin Amore è un desiderio, è un foco, è un genio al bello.

Lo chieda a me, che ne conosco il danno, chi non sa cosa sia d'Amor l'impero; molti amanti, che il core han prigioniero, penano per amor, ma non lo sanno.

S'ei ti dà gelosia cessa l'inganno, se non sei corrisposto è mal leggiero, poiché scossi dal giogo alma e pensiero la legge schermirai del gran tiranno.

Se ti spiace il tacer, palesa il core, se la meta tropp'alta empie la spene, o scordati d'amare, o cangia amore.

Se sei lontano han termine le pene; ma l'esser corrisposto c̀ il mal peggiore, perché scioglier non sai dolci catene. 
X.

Di vaga, e dolce bocca a' bei coralli soavi bissi, e porpore famose ove perdon le spine aspri intervalli, ed in perpetuo April regnan le rose.

D'un tesoro di perle argini, e valli ove la gioia il trono suo ripose; rendonsi in voi degni di scusa $\mathrm{i}$ falli, mentre d'Amor il nido in voi s'ascose.

Son sangue d'olocausti i tuoi colori, e pur se parli, o taci, amato labro svegli il cieco desire a dolci errori.

O gemello ridente, almo Cinabro, fabrica di rubino, urna de' cori, baci non voglio, e in te contemplo il fabro.

\section{1}

Z.

\section{Moralità d'amante}

B. F. Q. X.

Come manca la rosa in seno al prato cosí mancano l'ore al viver mio; cosí perde lo stral l'Arciero ingrato, cosí manca lo scopo al cieco Dio.

Veggio cader le penne al Nume alato, smorzano i molli ardori onde d'oblio; e al passo che l'età cangia lo stato, l'umana volontà cangia desio.

Mancan gli spirti piú vivaci, e chiusa già la mia mente in sé par che disarmi l'idea mendace, che d'error l'accusa. Guerra, guerra ad Amor, mio core a l'armi; già per lui manca l'estro a la mia Musa, come mancan le lettere ai miei carmi.

Si dedicano alla med. Sig. ${ }^{\text {ra }}$ D. Angela Voglia le seguenti rime. *

Già leggesti i miei carmi, ove scolpita si rimira l'idea di varij amori;

a nuove fole o bella ora t'invita 
Genio, che ti consacra i suoi sudori.

Benché dal ciglio tuo cercano vita

di Clio raminga $\mathrm{i}$ mal concetti ardori, gloria non bramo, io ti dimando aita, ombra ti chieggio, e non pretendo allori.

Leggi, c dove il vedrai, d'incauti affetti scusa, c difendi il debole trascorso onde gl'errori miei saran diletti.

E se al Pegaso mio rallento il morso, senza legge dircea, solo rifletti al desio de la meta, e non al corso.

* Dopo l'ultima poesia lipogrammatica dei Sonetti amorosi, questa dedica apre la collana intitolata Capricci, di cui si trascrive il sonetto introduttivo.

\section{NOTE}

1 Pagano e Bianchi 146. Si veda anche il Grove. Sub voce "Paglia" si legge soltanto: "PAGLIA, Francesco Maria: See Scarlatti (1,6 libs)."

2 Per un elenco completo di queste composizioni, si veda il Catalogo di Pagano e Bianchi, passim.

3 Per questi ed altri dati pertinenti, si veda Curtius 282-83; Boissonade 370 ss. Breve ed essenziale, ma illuminante per la storia e la natura del lipogramma, si rivela la trattazione di Pozzi 109-110.

4 Per una precisa definizione dei due termini, "lipogramma" e "lipogrammatico," si veda Battaglia, ad vocem.

\section{OPERE CONSULTATE}

BATTAGLIA, Salvatore. Grande dizionario della lingua italiana. Volume IX. Torino: UTET, 1975.

BOISSONADE, J. F. Critique littéraire sous le Premier Empire. Tome Premier. Paris: Librairie Académique Didier et C. ${ }^{\mathrm{e}}, 1863$.

CURTIUS, E. R. European Literature and the Latin Middle Ages. Princeton: Princelon U P, 1973.

Grove Dictionary of Music and Musicians. Voll. 10. New York: St. Martin's Press Inc., 1954-61.

PAGANO, Roberto e Lino BIANCHI. Alessandro Scarlatti. Catalogo generale delle opere, a cura di Giancarlo Rostirolla. Torino: ERI, Edizioni RAI Radiotelevisione Italiana, 1972. 
POZZI, Giovanni. Poesia per gioco. Prontuario di figure artificiose. Bologna: Il Mulino, 1984. 